\title{
Local Conformal Symmetry in Physics and Cosmology
}

\author{
Itzhak Bars \\ Department of Physics and Astronomy, \\ University of Southern California, Los Angeles, CA, 90089-0484, USA, \\ Paul Steinhardt \\ Physics Department, Princeton University, Princeton NJ08544, USA, \\ Neil Turok \\ Perimeter Institute for Theoretical Physics, Waterloo, ON N2L 2Y5, Canada.
}

(Dated: July 7, 2013)

\begin{abstract}
We show how to lift a generic non-scale invariant action in Einstein frame into a locally conformally-invariant (or Weyl-invariant) theory and present a new general form for Lagrangians consistent with Weyl symmetry. Advantages of such a conformally invariant formulation of particle physics and gravity include the possibility of constructing geodesically complete cosmologies. We present a conformal-invariant version of the standard model coupled to gravity, and show how Weyl symmetry may be used to obtain unprecedented analytic control over its cosmological solutions. Within this new framework, generic FRW cosmologies are geodesically complete through a series of big crunch - big bang transitions. We discuss a new scenario of cosmic evolution driven by the Higgs field in a "minimal" conformal standard model, in which there is no new physics beyond the standard model at low energies, and the current Higgs vacuum is metastable as indicated by the latest LHC data.
\end{abstract}




\section{Contents}

I. Why Conformal Symmetry?

II. Why Local Conformal Symmetry?

A. Global scale invariance

B. Local scale invariance

III. General Weyl Symmetric Theory
A. Gravity
B. Supergravity

15

IV. Conformal Cosmology 


\section{WHY CONFORMAL SYMMETRY?}

Scale invariance is a well-known symmetry [1] that has been studied in many physical contexts. A strong physical motivation for incorporating scale symmetry in fundamental physics [2] [3] [4] comes from low energy particle physics. Namely, the classical action of the standard model is already consistent with scale symmetry if the Higgs mass term is dropped. This invites the idea, which many have considered, that the mass term may emerge from the vacuum expectation value of an additional scalar field $\phi(x)$ in a fully scale invariant theory. Another striking hint of scale symmetry occurs on cosmic scales: the (nearly) scale invariant spectrum of primordial fluctuations, as measured by WMAP and the Planck satellite [5, 6]. This amazing simplicity seems to cry out for an explanation in terms of a fundamental symmetry in nature, rather than as just the outcome of a scalar field evolving along some particular potential given some particular initial condition.

In this paper, we consider the incorporation into fundamental physics of local conformal symmetry (or Weyl-symmetry): that is, classical local scaling symmetry in an action that includes the standard model coupled to gravity. A new result of our approach [7]-[12] is that using conformal symmetry we are able to solve the classical FRW equations across big crunch-big bang transitions, thus obtaining the full set of geodesically complete cosmological solutions of our conformal standard model given in Eq.(15). This follows from the new properties of the standard theory whose couplings to gravity includes all patches of field space that are required for geodesic completeness of all cosmological solutions for all times and any set of initial conditions ${ }^{1}$.

The models we describe contain no mass scales - no gravitational constant, no mass for the Higgs field, no cosmological constant and no mass parameters for the quarks, leptons or gauge bosons. All of these are prevented by the local conformal symmetry combined with the $\mathrm{SU}(3) \times \mathrm{SU}(2) \times \mathrm{U}(1)$ gauge symmetry of the standard model. There is only one source of mass which follows from gauge fixing (in some sense spontaneously breaking) the Weyl symmetry through a scalar field which is a singlet under $\mathrm{SU}(3) \times \mathrm{SU}(2) \times \mathrm{U}(1)$ or a combi-

\footnotetext{
${ }^{1}$ In this paper, we use the term "geodesic completeness" to refer to two notions: (a) geodesic continuation through all singularities separating patches of spacetime; and (b) avoidance of unnatural initial conditions by requiring infinite action for geodesics that reach arbitrarily far in the past. The two notions are inequivalent since one is local in time and the other is a global condition. Both properties are satisfied in our conformal standard model in the form given in Eq.(5) as described in section (IV).
} 
nation of both $\mathrm{SU}(3) \times \mathrm{SU}(2) \times \mathrm{U}(1)$ singlet and non-singlet fields, depending on the choice of gauge. This source, which is associated with the emergence of the Planck scale, drives the spontaneous breakdown of the electroweak symmetry, which in turn generates the other known masses of elementary constituents, with ratios of masses related to dimensionless constants.

Our approach to conformal symmetry has new features with significant physical consequences that were not considered before. While we shall work in $3+1$ dimensions throughout, there is a close connection with field theories and gravity or supergravity in $4+2$ dimensions in the context of 2T-physics [13]-[18] as will be pointed out occasionally throughout this paper. Stated directly in $3+1$ dimensions, some of the novel features and their consequences are as follows:

- In section (III) we provide the most general form of coupling gravity or supergravity with any number of scalar fields, fermions and gauge bosons, while maintaining local conformal symmetry. Only a specialized form of our general formalism in section (III) coincides with previously known methods for local conformal symmetry. We find that in a gauge symmetric theory, if there is only one physical scalar field (for example, the Higgs field) after all gauge choices are exhausted, then there is a unique gaugeinvariant and Weyl-invariant form for the Lagrangian representing the geodesically complete coupling to gravity.

- The minimal, realistic and locally conformal standard model coupled to gravity includes the Higgs doublet $H(x)$ and an additional $\mathrm{SU}(3) \times \mathrm{SU}(2) \times \mathrm{U}(1)$ singlet $\phi(x)$. Taking into account all of the local symmetry, there is a single physical scalar field identifiable with the observed Higgs field. Therefore, according to the statement above, this is a geodesically complete conformal standad model provided it utilizes our unique coupling to gravity as shown in Eq.(5) and as proven in section (IIIA). The relative minus sign between $\phi$ and $H$ that appears in this action is obligatory and is related to geodesic completeness as will be explained later. The unique structure of local scale symmetry has led to full analytic control of cosmological solutions and, in certain limits, enabled us to obtain the complete set of homogeneous cosmological solutions for all possible initial conditions of the relevant fields. This is discussed in section (IV).

- In a particular gauge of the spontaneously broken local scale symmetry (called c- 
gauge) we recover the usual renormalizable field theory of the standard model in the flat space limit, including a mass scale for the Higgs. In this gauge, low energy physics of our model coincides with the familiar form of the standard model unless more fields are included beyond those already observed, such as right handed neutrinos and dark matter which can be accommodated consistently with conformal symmetry following our methods. Thus, low energy physics at LHC scales is not sensitive to the conformal structures suggested in our approach.

- On the other hand, physics at cosmological scales can be quite sensitive to the conformal structures discussed in this paper as becomes apparent in certain gauges. In section (IV) we outline some of the phenomena that follow from our unique conformal coupling. In the minimal conformal standard model of Eq.(5), guided by our analytic solutions (supplemented, where necessary, with numerical methods), we propose a cyclic conformal cosmology driven only by the Higgs field with no recourse to other scalar fields such as an inflaton. The Higgs-driven cosmological scenario we propose is strongly motivated by the metastability of the Higgs vacuum as implied by the renormalization-group flow of the minimal standard model to large Higgs vev, with its parameters fixed by the LHC measurements [19].

The main discussion in this paper is at the classical field theory level, but it is worth commenting briefly on the important question of quantum corrections. There are two enormous hierarchies in the standard model coupled to gravity: the weak scale is around $10^{-16}$ and the dark energy scale is around $10^{-30}$ of the Planck scale. It is hard to understand how such tiny numbers enter fundamental physics, and why quantum corrections would not spoil these fine tunings.

In both cases, conformal symmetry has been suggested as a solution. We will not explore this here in any detail, except to note that the stability of such hierarchies in the perturbative standard model, which is what attracted attention in the past for the Higgs field, should not be in jeopardy from quantum corrections since dimensionless constants in a conformal theory are logarithmically divergent as opposed to the quadratic divergence of a bare Higgs mass term. To fully grasp how this can work at the quantum level for fundamental scalars and in particular the Higgs, requires a better understanding of how to perform regularization and renormalization consistent with local conformal symmetry in $3+1$ dimensions. Elabo- 
rating on earlier suggestions [4] [20] [21], there has been recent progress [22] in developing a renormalization theory consistent with Weyl symmetry. According to this new work, local conformal symmetry remains as a valid symmetry at the quantum level as anticipated in [4]. The local scale invariance survives quantization even though there is a trace anomaly in the stress tensor of the matter sector of the theory; this point that caused confusion in the past is clarified by noting that the trace of the matter stress tensor is distinct from the generator of local scale transformations that includes the additional fields, such as $\phi$, that implement and establish the local conformal symmetry. In passing, we note that if $3+1$ dimensional conformal symmetry is treated consistently within 2T-physics in $4+2$ dimensions, then scale (dilation) symmetry is a part of a linearly realized $\mathrm{SO}(4,2)$ in the flat limit which is presumably not anomalous just as its Lorentz subgroup $\mathrm{SO}(3,1)$ in $3+1$ dimensions cannot be anomalous. This quantum aspect of 2 T-physics is under investigation directly in $4+2$ dimensions and this is expected to shed additional light on these issues.

A plan of this paper is as follows. In Sec. II, we will review and expand on the motivation for global and local symmetry and show how to recast (or "lift") the standard model plus gravity into a locally gauge invariant, Weyl- symmetric theory by adding new fields and, at the same time, introducing compensating gauge symmetries, in such a way that the theory is both Weyl invariant and geodesically complete. The gauge fixed version of this theory reverts back to the familiar minimal form of the standard model at low energies wherever gravity is negligible. However, in regions of spacetime where strong gravity effects are important, such as in cosmology, especially close to the singularity, the geodesically complete Weyl lifted version plays a crucial role, as described in Sec. IV.

In section III, following [18] we present a general form for a Lagrangian consistent with Weyl symmetry for any number of scalar fields. We argue that, if the Weyl invariant theory is equivalent to having only a single physical scalar field after gauge fixing (like the physical Higgs), then by field redefinitions it is always possible to recast the theory into our version given in Eq.(51) which includes all patches in field space so as to insure geodesic completeness.

As another example of our general formalism, we lift the Bezrukov-Shaposhnikov Higgs inflation model [23] to a fully scale invariant model, making it logically and physically consistent. Based on our formalism for constructing general Weyl-invariant actions, we show that the model is not unique. In fact, there are many single-field cosmological Higgs models with the same properties, all consistent with conformal symmetry and producing the 
same inflationary outcome. However, this inflationary scenario is not geodesically complete, furthermore, in view of our complete set of solutions, it is extremely unlikely.

In Sec. IV, we turn more generally to cosmology. We explain that a Weyl-lifted theory can resolve the singularity and enable the geodesic completion of cosmological spacetimes, while also indicating the presence of new phenomena. Our previous work in [7]-[12] elaborated in some detail on the properties of geodesically complete cosmological analytic solutions in the context of solvable examples of what was supposed to be toy models. However the same models now re-appear in our conformal standard model and hence our previous solutions are now the full set of cosmological solutions for all initial conditions of the familiar standard model. In particular, we point out a model independent attractor mechanism discovered in [10] that may help to determine the likely initial conditions of our universe just after the big bang. The Weyl-invariant formulation also provides a natural framework for incorporating cyclic cosmology [24, 25] driven only by the Higgs field as introduced. In the case that the Higgs field is metastable, the Higgs inflation model is inoperable, but the minimal singleHiggs model is naturally compatible with the cyclic picture [26]. In general, the Weylinvariant approach also hints at the intriguing possibility that the minimal, electroweak Higgs may have played a central role in cosmic evolution as explained in detail in [27].

There is a deep connection between the ideas presented in this paper and theories in 4-space and 2-time dimensions. Many of the ideas discussed here for a global or local conformally symmetric theory emerged progressively from developing the $4+2$ dimensional formalism since 1996 (for a recent overview see [13]), and then for the standard model as given in [14], for gravity in [16], for SUSY in [17] and supergravity in [18]. The formulation given in [18], with many scalars coupled to gravity and supergravity in $4+2$ and $3+$ 1 dimensions was the precursor of the general formalism presented in section III, while the simplest specific models were analyzed cosmologically in some detail in [7]-[12]. There are some recent similar examples [28] that overlap with our conformal symmetry vision in $3+1$ dimensions; these seem to be oblivious to our previous publications that introduced at an earlier stage the crucial conformal structures with restrictions on scalars in $3+1$ dimensions as predictions from 2T-physics [18]. The $4+2$ theory has more predictions of hidden symmetries and dualities in $3+1$ dimensions which are mainly understood in classical and quantum mechanical contexts [13] and are also partially developed in field theory [29]; these go well beyond conformal symmetry in their implications for unification 
and the meaning of spacetime and are bound to play an interesting role in future progress.

We emphasize that, despite the name, the physics content in the 2T-physics formalism in $(d+2)$ dimensions is same as the physics content in the standard 1T-physics formalism in $(d-1)+1$ dimensions except that $2 \mathrm{~T}$ physics provides a holographic perspective and, due to a much larger set of gauge symmetries, naturally makes predictions that are not anticipated in 1T-physics. These additional gauge symmetries are in phase space rather than position space, and can be realized only if the formalism is developed with two times. Nevertheless, the gauge invariant sector of $2 \mathrm{~T}$-physics is equivalent to a causal 1-time spacetime without any ghosts.

In this paper, we will stick to $3+1$ dimensions, advocating a coherent overall picture of a conformally invariant formulation of fundamental physics and cosmology. However, we will occasionally remind the reader that these outcomes naturally follow from $4+2$ dimensions with appropriate (but unusual) gauge symmetries.

\section{WHY LOCAL CONFORMAL SYMMETRY?}

In this section, we will describe the motivation for and construction of simple theories with global and local scale invariance. An important application is the lift of the standard model plus gravity into a Weyl- invariant theory that is also geodesically complete.

\section{A. Global scale invariance}

We begin by examining a scale invariant extension of the standard model of particles and forces. For example consider the usual standard model with all the usual fields, including the doublet Higgs field $H(x)$ coupled to gauge bosons and fermions, but add also an $\mathrm{SU}(2) \times \mathrm{U}(1)$ singlet $\phi(x)$ (plus right handed neutrinos and dark matter candidates), and take the following purely quartic renormalizable potential involving only the minimal set of scalar fields

$$
V(H, \phi)=\frac{\lambda}{4}\left(H^{\dagger} H-\alpha^{2} \phi^{2}\right)^{2}+\frac{\lambda^{\prime}}{4} \phi^{4}
$$

This model, discussed at some length in section VI of [14], is the minimal extension of the standard model that is fully scale invariant at the classical level, globally. See also [30]-[36] that use a similar field, and the different approach that also adds a Weyl vector field [37] 
leading to different physical consequences. The field $\phi(x)$, which we call the "dilaton", absorbs the scale transformations and is analogous to the dilaton in string theory. In the current context, it has a number of interesting features: Due to $\mathrm{SU}(3) \times \mathrm{SU}(2) \times \mathrm{U}(1)$ gauge symmetry, the singlet $\phi$ is prevented from coupling to all other fields of the Standard model - except for the additional right handed singlet neutrinos or dark matter candidates. These features of $\phi$, that prevent it from interacting substantially with standard visible matter except via the Higgs in Eq.(1), suggest naturally that $\phi$ itself could be a candidate for dark matter [14].

The only parameters associated with $\phi$, that are relevant for our discussion, are $\alpha$ and $\lambda^{\prime}$. For positive $\lambda^{\prime}$ the minimum of this potential occurs at $H^{\dagger} H=\alpha^{2} \phi^{2}$. Accordingly, the vacuum expectation value of the Higgs may fluctuate throughout spacetime, depending on the dynamics of $\phi(x)$, without breaking the scale symmetry. However, if for some reason (e.g. driven by quantum fluctuations or gravitational interactions) $\phi$ develops a vacuum expectation value $\phi_{0}$ which is constant in some region of spacetime, then the Higgs is dominated by a constant vacuum expectation value

$$
H_{0}^{\dagger} H_{0}=\alpha^{2} \phi_{0}^{2} \equiv \frac{v^{2}}{2}
$$

with $v$ fixed by observation to be approximately $246 \mathrm{GeV}$. The Higgs vacuum $H_{0}$ provides the source of mass for all known elementary forms of matter, quarks, leptons, and gauge bosons (while $\phi_{0}$ may be the source of Majorana mass for neutrinos). The observation at the LHC of the Higgs particle, which is just the small fluctuation on top of the vacuum value $v$, has by now solidified the view that this is how nature works in our region of the universe, at least up to the energy scales of the LHC.

Fig. 1 illustrates how the Higgs field slowly relaxes to the spontaneously broken symmetry vacuum described by Eq. (2) beginning from large oscillations shortly after the big bang. Here, as is the case throughout the paper, the solutions are in the limit of negligible gauge and top quark mass coupling so that the Higgs evolution is described by classical equations of motion. This time-dependent behavior of the Higgs is driven by the evolution of the field $\phi$, as anticipated in [15] for this simple model. In fact, it is realized as the generic solution for all homogeneous cosmological solutions if the Higgs vacuum is stable.

There are other interesting theoretical structures worth noting about this scale invariant setup. Instead of supersymmetry, conformal symmetry may explain the stability of the 
hierarchy between the low mass scale of $246 \mathrm{GeV}$ versus the Planck scale of $10^{19} \mathrm{GeV}$, as suggested in [15]. The conformal protection of the hierarchy is not as clear-cut as SUSY's protection and requires better understanding of regularization and renormalization techniques consistent with conformal symmetry as outlined in the introduction.

What about the dilaton (fluctuations in $\phi$ ) that emerges in the broken scale invariance scenario above? At least from the perspective of only the standard model, the dilaton is a massless Goldstone boson due to the spontaneous breaking of the global scale invariance. As discussed in some detail in section VI of [14], the original doublet field $H$ is the only field coupled to known matter, while $\phi$ is decoupled. However, after the spontaneous breaking, $H$ must be rewritten as a mixture of the mass eigenstates of the model, which include the observed massive Higgs particle and the massless dilaton (or maybe low-mass dilaton if the scale symmetry is broken by some source). The mixing strength is controlled by the dimensionless parameter

$$
\alpha=(246 G e V) /\left(\sqrt{2} \phi_{0}\right),
$$

that appears in Eq.(2). Therefore, through this mixing, the (pseudo-) Goldstone dilaton must couple to all matter, just like the Higgs does, namely with a coupling given by the mixing angle $\left(\sin (\theta)=\alpha /\left(1+\alpha^{2}\right)^{1 / 2}\right)$ times the mass of the particle divided by $v$. The largest coupling is to the top quark. If $\phi_{0}$ is much larger than $246 \mathrm{GeV}$, it is possible for the dilaton to hide from accelerator experiments due to the weak coupling of $\alpha$ in Eq.(3).

Such a (pseudo) Goldstone boson has many other observable consequences, including a long range force that competes with gravity and contributions to quantum effects as a virtual particle in Feynman loop diagrams. If such an additional massless (or low mass scalar) is observed in experiments, it would be strong evidence in favor of the scale invariant scenario. However, if it is not observed, this could be interpreted merely as setting a lower limit on the scale $\phi_{0}$.

Eventually it must be understood what sets the scale $\phi_{0}$. The model as presented above has no mechanism at the classical level to set the scale $\phi_{0}$; its equations of motion are self consistent at the minimum of the potential, $H_{0}^{\dagger} H_{0}=\alpha^{2} \phi_{0}^{2}$, only if $\lambda^{\prime}=0[14]$; then $\phi_{0}$ remains undetermined due to a flat direction in the remaining term in the potential (11). The value for $\phi_{0}$ would then be obtained phenomenologically from experiment, without a theoretical explanation. Quantum corrections, such as those discussed in [39], may alleviate this problem by removing the flat direction. However, if the quantum corrections are small, 
there is the danger that $\phi_{0}$ would be so low that it is not possible to obtain the small value of $\alpha$ in Eq.(3) required to protect the dilaton from current experimental limits.

\section{B. Local scale invariance}

Since at present there is no sign of the dilaton in low energy physics, suppose it does not exist at all as a degree of freedom. Is this incompatible with the idea that conformal symmetry underlies fundamental physics? Not at all, because any possible phenomenological problems associated with a Goldstone boson fluctuation of $\phi$ can be overcome if the scaling symmetry is a local gauge symmetry, known as the Weyl symmetry. Then the massless fluctuations of the dilaton can be eliminated by fixing a unitary gauge ${ }^{2}$.

The standard model decoupled from gravity has no local scale symmetry that could remove a Goldstone dilaton. But such a gauge symmetry can in fact be successfully incorporated as part of the standard model provided it is coupled to gravity in the right way. Coupling the standard model to Einstein gravity in the conventional way makes no sense because the dimensionful Newton constant explicitly breaks scale invariance. If scale invariance is already broken in one sector of the theory, then there is no rationale for requiring that it be a good symmetry in another part of the theory. At best, it would occur as an accidental symmetry of low energy physics and only when gravity is negligible. This is not the scenario we have in mind; we argue for a fully scale invariant approach to all physics, a natural outcome of the larger gauge symmetries in $4+2$ dimensions, as formulated in 2Tphysics. Happily, the idea of an underlying $4+2$ dimensions with appropriate extra gauge symmetry fits all known physics in $3+1$ dimensions, from dynamics of particles and field theory [13]-[17] all the way to supergravity [18]. So, consistency with this larger underlying structure is well motivated.

In fact, there is a locally scale invariant field theory in $3+1$ dimensions, compatible with 2T-physics in $4+2$ dimensions, that couples the standard model and gravity with no dimensionful constants. We do not mean conformal gravity which has ghost problems, but

\footnotetext{
${ }^{2}$ The interesting features of the dilaton of the previous section, including massive fluctuations, could emerge from one more $\mathrm{SU}(2) \times \mathrm{U}(1)$ scalar field as part of the general theory that we will discuss in section (IIIA). But for simplicity in this section we will first concentrate on the minimal model that contains only the confirmed observed degrees of freedom up to now, thus allowing the fluctuations of a single $\phi$ to be eliminated by a gauge symmetry in this minimal model.
} 
rather the non-minimal conformal coupling of the curvature $R(g)$ to scalar fields [3] which is invariant under Weyl transformations as a gauge symmetry. In the next section we will discuss a generalized Weyl invariant coupling with many scalar fields also predicted by 2Tphysics, but in this section we begin with the well known method of conformally coupled scalars, as follows [3] [4] ,

$$
\frac{1}{12} \phi^{2} R(g)+\frac{1}{2} g^{\mu \nu} \partial_{\mu} \phi \partial_{\nu} \phi
$$

These two terms form an invariant unit (up to a total derivative) under local scale transformations, $g_{\mu \nu} \rightarrow \Omega^{-2} g_{\mu \nu}, \phi \rightarrow \Omega \phi$, with a local parameter $\Omega(x)$. When there are more scalar fields, the most general way of achieving local conformal symmetry is discussed in section (10). However, when there is only one additional scalar field beyond $\phi$, which is in a single representation of a Yang-Mills gauge group, there is a unique way to also achieve geodesic completeness as explained in more detail in sections (IIIA|IV). Hence, for geodesic completeness we require that not only the field $\phi$, but also the doublet Higgs field be a set of conformally coupled scalars consistent with $\mathrm{SU}(2) \times \mathrm{U}(1)$. Namely using the unit $\frac{1}{6}\left(H^{\dagger} H\right) R(g)+g^{\mu \nu} D_{\mu} H^{\dagger} D_{\nu} H$ which is also locally invariant (up to a total derivative), we can lift the globally scale invariant standard model of the last section into a locally invariant one, while also being coupled to gravity in a gedesically complete theory. All other terms present in the usual standard model, namely all fermion, gauge boson and Yukawa terms, when minimally coupled to gravity are already automatically invariant under the local Weyl symmetry.

Hence a Weyl invariant action $S=\int d^{4} x \mathcal{L}(x)$ that describes the coupling of gravity and the standard model is given by

$$
\mathcal{L}(x)=\sqrt{-g}\left[\begin{array}{c}
\frac{1}{12}\left(\phi^{2}-2 H^{\dagger} H\right) R(g) \\
+g^{\mu \nu}\left(\frac{1}{2} \partial_{\mu} \phi \partial_{\nu} \phi-D_{\mu} H^{\dagger} D_{\nu} H\right) \\
-\left(\frac{\lambda}{4}\left(H^{\dagger} H-\alpha^{2} \phi^{2}\right)^{2}+\frac{\lambda^{\prime}}{4} \phi^{4}\right) \\
+L_{\mathrm{SM}}\left(\begin{array}{c}
\text { quarks, leptons , gauge bosons, } \\
\text { Yukawa couplings to } H \& \phi, \text { dark matter. }
\end{array}\right)
\end{array}\right]
$$

The only Yukawa couplings of $\phi$ allowed by $\mathrm{SU}(3) \times \mathrm{SU}(2) \times \mathrm{U}(1)$ are to the right handed neutrinos for which it becomes a source of mass. Note the relative minus sign between $\phi$ and $H$ terms, which is required, as explained below. Here $L_{\mathrm{SM}}$ is the well known standard model Lagrangian minimally coupled to gravity, except for the Higgs kinetic and potential 
terms, which are now modified and explicitly written out in the first three lines. This action is invariant under Weyl rescaling with an arbitrary local parameter $\Omega(x)$ as follows

$$
\begin{gathered}
g_{\mu \nu} \rightarrow \Omega^{-2} g_{\mu \nu}, \phi \rightarrow \Omega \phi, H \rightarrow \Omega H, \\
\psi_{q, l} \rightarrow \Omega^{3 / 2} \psi_{q, l}, A_{\mu}^{\gamma, W, Z, g} \rightarrow \Omega^{0} A_{\mu}^{\gamma, W, Z, g},
\end{gathered}
$$

where $\psi_{q, l}$ are the fermionic fields for quarks or leptons, and $A_{\mu}^{\gamma, W, Z, g}$ are the gauge fields for the photon, gluons, $W^{ \pm}$and $Z$. Note that the gauge bosons do not change under the Weyl rescaling.

We note that the Lagrangian (5) is the one obtained in the second reference in [16] from the $4+2$ version of the standard model [14] [15] coupled to 2T-gravity [16], by a method of gauge fixing and solving some kinematical equations associated with constraints related to the underlying gauge symmetries. In that approach we learned that the Weyl symmetry is not an option in $3+1$ dimensions, it is a prediction of 2T-physics: the $4+2$ dimensional theory is not Weyl invariant, but yet the local Weyl symmetry in $3+1$ dimensions emerges as a remnant gauge symmetry associated with the general coordinate transformations as they act in the extra $1+1$ dimensions. So the Weyl symmetry is a required symmetry in $3+1$ dimensions as predicted in the $4+2$ dimensional approach; this symmetry carries information and imposes properties related to the extra $1+1$ space and time dimensions [13].

The Weyl gauge symmetry of the action in (5) does not allow any dimensionful constants: no mass term in the Higgs potential, no Einstein-Hilbert term with its dimensionful Newton constant, or any other mass terms. This is a very appealing starting point because it leads to the emergence of all dimensionful parameters from a single source. That source is the field $\phi(x)$ that motivated this discussion in the previous section, and the only scale is then generated by gauge fixing $\phi$ to a constant for all spacetime

$$
\phi(x) \rightarrow \phi_{0}
$$

In the gauge fixed version of the Lagrangian where $\phi(x) \rightarrow \phi_{0}$, we can express the physically important dimensionful parameters, namely, the Newton constant $G$, the cosmological constant $\Lambda$ associated with dark energy, and the electro-weak scale $v$, in terms of $\phi_{0}$ :

$$
\frac{1}{16 \pi G}=\frac{\phi_{0}^{2}}{12}, \frac{\Lambda}{16 \pi G}=\lambda^{\prime} \phi_{0}^{4}, H_{0}^{\dagger} H_{0}=\alpha^{2} \phi_{0}^{2} \equiv \frac{v^{2}}{2} .
$$


Since the field $\phi(x)$ in this gauge ceases to be a degree of freedom altogether, the massless dilaton is absent and the potential problem with global scaling symmetry is avoided. Nevertheless, there still is an underlying hidden conformal symmetry for the full theory.

We can explain now why it is necessary to have the opposite signs for $\phi$ and $H$ in the first two lines of the action in Eq.(5). The positive sign for $\phi$ is necessary in order to obtain a positive gravitational constant in (8). However, conformal symmetry then forces the kinetic term for $\phi$ to have the wrong sign, so $\phi$ is a ghost. This can be seen to be a gauge artifact, though, since in a unitary gauge the ghost $\phi$ is fixed to a constant or expressed in terms of other degrees of freedom. This also explains why $H$ must have the opposite sign, since otherwise $H$ would be a real ghost. This relative sign has important consequences in cosmology as follows.

In flat space $R(g) \rightarrow 0$, where experiments such as those at the LHC are conducted, the Lagrangian above becomes precisely the usual standard model, including the familiar tachyonic mass term for the Higgs. Furthermore, in weak gravitational fields, at low energies, the gravitational effect of the Higgs field coupling to the curvature, $\frac{1}{12}\left(\phi_{0}^{2}-2 H^{\dagger} H\right) R(g)$, is ignorable since $H$ (order of $v \approx 246 \mathrm{GeV}$ ) is tiny compared to the Planck scale $\left(\phi_{0} \approx 10^{19}\right.$ $\mathrm{GeV}$ ). Actually, the gravitational constant measured at low energies is corrected by the electroweak scale, namely $(16 \pi G)^{-1}=\left(\phi_{0}^{2}-v^{2}\right) / 12$ instead of (요), but in practice this is a negligible correction since $v^{2} \ll \phi_{0}^{2}$. So, at low energies there is no discernible difference between our Weyl-lifted theory and the usual standard model. The practically isolated standard model appears as a renormalizable theory decoupled from gravity.

However, in the cosmological context, the conformally coupled $H$ can and will be large at some stages in the evolution of the universe. Working out the dynamics of cosmological evolution, it turns out that, for generic initial conditions, $H^{\dagger} H$ typically grows to large scales over cosmological times, hitting $\left(\phi_{0}^{2}-2 H^{\dagger} H\right) \rightarrow 0$ in the vicinity of a big bang or big crunch singularity. This behavior plays an essential role in cosmological evolution, as well as in the resolution of the singularity via geodesic completeness. In our conformal theory, the standard model is not isolated from gravity, and we posit that the Higgs field can play a bigger role in nature than originally anticipated. 
We may take the Higgs doublet in the unitary gauge of the $\mathrm{SU}(2) \times \mathrm{U}(1)$ gauge symmetry

$$
H(x)=\left(\begin{array}{c}
0 \\
\frac{1}{\sqrt{2}} s(x)
\end{array}\right) .
$$

The field $s(x)=v+\delta h(x)$, where $\delta h(x)$ is the Higgs field fluctuation on top of the electroweak vacuum, is then identified with the generic field $s(x)$ that appeared in our cosmological papers [7]-[12]. The action for $s(x)$ has the exact same form in those papers as here.

Thus, our previous analytical cosmological solutions can now be applied to investigate the cosmological properties of the Higgs field. When the parameter $\alpha$ vanishes, our previous work provides the full set of geodesically complete analytic cosmological solutions for all initial conditions of the relevant fields in the standard model coupled to gravity. The non-zero $\alpha$ is then easily taken into account with numerical methods. This much analytic control over cosmological properties of a realistic theory is unprecedented. This became a very valuable tool that led us into a new cosmological scenario driven only by the Higgs field, as outlined in section (IV]) and completed in [27].

\section{GENERAL WEYL SYMMETRIC THEORY}

The gauge symmetries derived from 2T-gravity and 2T-supergravity in $4+2$ dimensions lead to the general Weyl invariant coupling described below for any number of scalar fields in $3+1$ dimensions [18]. This generalizes the possible forms of conformally coupled scalar theories beyond those encompassed by Eq.(4) and allows for richer possibilities for model building consistent with local scale invariance. We ignore the spinors and gauge bosons whose minimal couplings to gravity are already automatically Weyl symmetric.

\section{A. Gravity}

We begin with gravity without supersymmetry. In the next subsection we will indicate the additional constraints that emerge in supergravity. We assume any number of real scalar fields $\phi^{i}(x)$ labeled by the index $i$. If there are complex fields, we can extract their real and imaginary parts and treat those as part of the $\phi^{i}$. We introduce a Weyl factor 
$U\left(\phi^{i}\right)$, a sigma-model-type metric in field space $G_{i j}\left(\phi^{i}\right)$ and a potential $V\left(\phi^{i}\right)$. The general Lagrangian takes the form

$$
\mathcal{L}=\sqrt{-g}\left(\frac{1}{12} U\left(\phi^{i}\right) R(g)-\frac{1}{2} G_{i j}\left(\phi^{i}\right) g^{\mu \nu} \partial_{\mu} \phi^{i} \partial_{\nu} \phi^{j}-V\left(\phi^{i}\right)\right) .
$$

The results given in [18] are the following constraints on these functions.

- $U\left(\phi^{i}\right)$ must be homogeneous of degree two, $U\left(t \phi^{i}\right)=t^{2} U\left(\phi^{i}\right)$; and $V\left(\phi^{i}\right)$ must be homogeneous of degree four, $V\left(t \phi^{i}\right)=t^{4} V\left(\phi^{i}\right)$; and $G_{i j}\left(\phi^{i}\right)$ must be homogeneous of degree zero, $G_{i j}\left(t \phi^{i}\right)=G_{i j}\left(\phi^{i}\right)$.

- The following differential constraints must also be satisfied. These may be interpreted as homothety conditions on the geometry in field space

$$
\partial_{i} U=-2 G_{i j} \phi^{j}, \quad \phi^{i} \partial_{i} U=2 U, G_{i j} \phi^{i} \phi^{j}=-U
$$

The second and third equations follow from the first one and the homogeneity requirements.

- Physics requirements also include that $G_{i j}$ cannot have more than one negative eigenvalue because the local scale symmetry is just enough to remove only one negative norm ghost. However, if more gauge symmetry that can remove more ghosts is incorporated, then the number of negative eigenvalues can increase accordingly. The gauged R-symmetry in supergravity (which is automatic in the $4+2$ approach) is such an example.

In [18] these rules emerged from gauge symmetries in 2T-gravity. Since Weyl symmetry is an automatic outcome from $4+2$ dimensions, we can check that these same requirements follow directly in $3+1$ dimensions by imposing Weyl symmetry on the general form in Eq.(10).

As an example, it is easy to check that all these conditions are automatically satisfied by the action given in Eq.(5) , with one $\phi$ and four real fields in the doublet $H$. Using the symbol $s$, as $s^{2} \equiv 2 H^{\dagger} H$, we write it in the form

$$
U=\phi^{2}-s^{2}, V=\phi^{4} f(s / \phi), G_{i j}=\eta_{i j},
$$


where $\eta_{i j}$ is a flat Minkowski metric in the 5 -dimensional field space with a single negative eigenvalue - this reduces to a 2-dimensional $\eta_{i j}$ in the unitary gauge of Eq.(9) since indeed there is only a single physical field $s$. In our work [7]-[12] generally we let the potential $f(z)$ to be an arbitrary function of its argument $z=s / \phi=\sqrt{2 H^{\dagger} H} / \phi$, as allowed by the Weyl symmetry conditions above. In Eq.(1) we have a purely quartic renormalizable potential, $V=\frac{\lambda}{4}\left(s^{2}-\alpha^{2} \phi^{2}\right)^{2}+\frac{\lambda^{\prime}}{4} \phi^{4}$. When quantum corrections are included, $f(z)$ contains logarithmic corrections, but by re-examining the underlying symmetry, the effective quantum potential can again be rewritten in the form $\phi^{4} f(s / \phi)$, consistent with the local conformal symmetry. We use the quantum corrected potential in our discussion of Higgs cosmology.

Next we give the general solution of the requirements above in a convenient parametrization for $n+1$ fields labelled with $i=0,1,2, \cdots, n$, namely, $\phi^{i}=\left(\phi, s^{I}\right)$, where $\phi^{0} \equiv \phi$ is distinguished, while $s^{I}$ with $I=1,2, \cdots, n$ are all the other scalar fields. Then the general solution to the conditions above for Weyl gauge symmetry takes the following form

$$
\begin{aligned}
U\left(\phi, s^{I}\right) & =\phi^{2} u(z), \text { with any } u\left(z^{I}\right), \\
V\left(\phi, s^{I}\right) & =\phi^{4} f(z), \text { with any } f\left(z^{I}\right), \\
G_{I J}\left(z^{I}\right) & =\text { any non-singular } n \times n \text { metric, } \\
G_{0 I}(z) & =G_{I 0}(z)=-\frac{1}{2}\left(\frac{\partial u}{\partial z^{I}}+2 G_{I K} z^{K}\right), \\
G_{00}(z) & =-u+z^{I} \frac{\partial u}{\partial z^{I}}+z^{I} z^{J} G_{I J},
\end{aligned}
$$

where the ratio $z^{I} \equiv s^{I} / \phi$ is gauge invariant. Thus, after using the chain rule, $\partial / \partial z^{I}=$ $\phi \partial / \partial s^{I}$, the general Weyl invariant action becomes

$$
\mathcal{L}=\sqrt{-g}\left(\begin{array}{c}
\frac{1}{12} U(\phi, s) R(g)-V(\phi, s) \\
+\frac{1}{2}\left(U-s^{I} \frac{\partial U}{\partial s^{I}}-s^{K} s^{L} G_{K L}\right)\left(\partial^{\mu} \ln \phi\right)\left(\partial_{\mu} \ln \phi\right) \\
-\frac{1}{2} G_{I J}\left(\partial^{\mu} s^{I} \partial_{\mu} s^{J}\right)+\left(2 G_{I J} s^{J}+\frac{\partial U}{\partial s^{I}}\right) \partial^{\mu} s^{I} \partial_{\mu} \ln \phi
\end{array}\right)
$$

with the $U, V, G_{I J}$ in Eqs.(13-15). It should be noted that $G_{I J}\left(z^{I}\right), u\left(z^{I}\right), f\left(z^{I}\right)$ are not determined by Weyl symmetry alone. Various other symmetries in a given model could restrict them. Any choice consistent with additional symmetries is permitted in the construction of physical models. For example, the model in Eq.(5) has the $\mathrm{SU}(3) \times \mathrm{SU}(2) \times \mathrm{U}(1)$ symmetry. In particular, local superconformal symmetry gives more severe restrictions by relating $G_{i j}$ and $U$ from the beginning, as discussed below. 
As the number of scalar fields increases, the restrictions imposed by Weyl symmetry become less severe. For example, an additional $\mathrm{SU}(3) \times \mathrm{SU}(2) \times \mathrm{U}(1)$ gauge singlet field beyond $\phi$ that would be needed to reproduce the phenomenology of the dilaton-like singlet discussed in section (IIA) can be included with slightly more freedom on its coupling parameters. Keeping such possibilities in mind for more general phenomenological considerations, we the define the minimal model to include only the standard Higgs doublet and the singlet $\phi$ as in the previous section.

Hence, for clarity we will write out the general Weyl invariant Lagrangian for the case of only the Higgs doublet field $H$ plus $\phi$. This generalizes Eq.(5), but we suppress the other fields in our discussion. Furthermore, we will work directly with the gauge fixed version of the Higgs field in Eq.(9), so the Higgs doublet is reduced to a single field $s(x)$ as in (99). In that case, from Eqs. (13) 17) we obtain

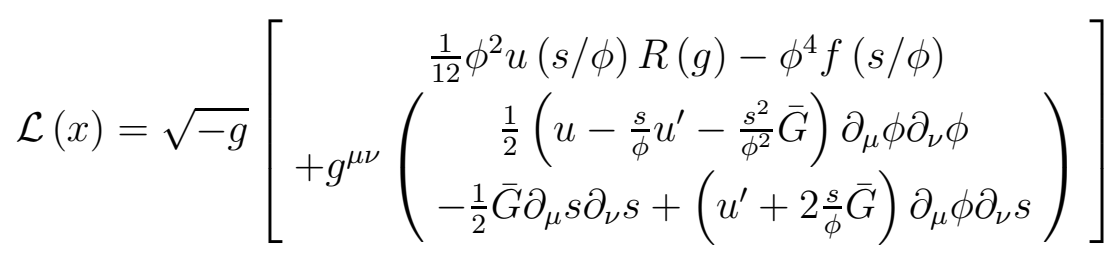

where $G_{I J}$ reduces to $G_{11}(s) \equiv \bar{G}(s)$ for the single field.

Generally, $\bar{G}(s / \phi), u(s / \phi), f(s / \phi)$ in Eq.(19) are three arbitrary functions of the Higgs field, $z=s / \phi=\sqrt{2 H H} / \phi$, which may be used for model building. As an example, if we take $U(\phi, s)=\phi^{2}+\xi s^{2}$, and $\bar{G}=1$, we obtain a relatively simple kinetic term with an arbitrary potential

$$
\mathcal{L}(x)=\sqrt{-g}\left[\begin{array}{c}
\frac{1}{12}\left(\phi^{2}+\xi s^{2}\right) R(g)-\phi^{4} f(s / \phi) \\
+\left(\begin{array}{c}
\frac{1}{2}\left(1-\frac{s^{2}}{\phi^{2}}(1+\xi)\right) \partial_{\mu} \phi \partial^{\mu} \phi \\
-\frac{1}{2} \partial_{\mu} s \partial_{\nu} s+2 \frac{s}{\phi}(1+\xi) \partial_{\mu} \phi \partial^{\mu} s
\end{array}\right)
\end{array}\right]
$$

This Lagrangian is Weyl invariant for any value of the constant parameter $\xi$, but we see now a simple generalization of the special simplifying role played by $\xi=-1$ that corresponds to the conformally coupled scalars of Eq.(1).

In the general single-s Lagrangian (19), field redefinitions of the gauge invariant variable $z \rightarrow F(z)$ (or $s \rightarrow \phi F(s / \phi)$ ) may be used to map one of these three functions, $\bar{G}(s / \phi), u(s / \phi), f(s / \phi)$, to any desired function of $z$ without changing the form of the Lagrangian in (19). For example it may be convenient to take $\bar{G}=1$, and still obtain all single-s Weyl-symmetric models by using all possible $u(z), f(z)$. Another option is to take 
all possible $\bar{G}(z), f(z)$ with a fixed $u(z)=1-z^{2}$ as in Eq.(5), which has certain advantages for understanding geodesic completeness [10] of all the fields $\phi, s, g_{\mu \nu}$, in cosmological spacetimes, as discussed in Sec. IV. If we choose $u(z)=1-z^{2}$ and demand renormalizability of the action in the limit of $\phi^{2} \gg s^{2}$ (where gravity effectively decouples, as seen in the gauge $\phi=\phi_{0}$ with $\phi_{0} \gg s$, we are forced to $\bar{G}=1$ and $f(z)$ being a quartic polynomial.

The last form is actually quite unique. We now give a proof that the general model of Eq.(19) with a single $s$ (which corresponds to our minimal realistic model) can always be written in the geodesically complete form that we advocated in our previous work [8]-[9] and implemented in proposing the action in Eq.(55). We begin in the notation of Eq.(10) with only two fields $\phi^{i}=(\phi, s)$ labelled by $i=0,1$. Using well known results of geometry in 2 dimensions, the metric in field space $G_{i j}(\phi)$ can always be diagonalized by general field reparametrizations (as in general relativity) and put into the conformally flat form $G_{i j}=$ $g(\phi) \eta_{i j}$ where $\eta_{i j}$ is the flat metric in 2 dimensions. Using further field reparametrizations with an overall rescaling consistent with Weyl transformations, the factor $g(\phi)$ can also be set equal to the constant $g(\phi)=1$. Having arrived at $G_{i j}=\eta_{i j}$ as still the most general metric in field space, the only possible form of $U\left(\phi^{i}\right)$ that is consistent with the local Weyl symmetry is $U\left(\phi^{i}\right)=\left(\phi^{2}-s^{2}\right) / 12$. This completes the general proof that the action in Eq.(5) is the most general Weyl invariant theory without losing generality. Then the complete analysis of homogeneous cosmological solutions provided in our previous work [8][9] shows that this form is the general geodesically complete version of the theory. Certain other forms for $U\left(\phi^{i}\right)$ arrived at by field reparametrizations, in particular purely positive forms of $U\left(\phi^{i}\right)$, always end up putting restrictions in field space inadvertently, and such specialized restrictions on fields is what leads to geodesic incompleteness.

Hence, the a class of well motivated models that we used in many of our studies amounts to $\bar{G}(s / \phi)=1, u(s / \phi)=1-s^{2} / \phi^{2}$, and the low energy renormalizable quartic potential $V(\phi, s)=\phi^{4} f(s / \phi)$, with $f(s / \phi)=\frac{\lambda}{4}\left(s^{2} / \phi^{2}-\alpha^{2}\right)^{2}+\frac{\lambda^{\prime}}{4}$. This is the model that now is identical to the conformally symmetric standard model we proposed. Its homogeneous cosmological equations have been solved analytically exactly in [11] for all possible initial conditions of the fields, including radiation and curvature, and all possible values of the parameters $\lambda, \lambda^{\prime}$, but with $\alpha=0$. After this much analytic control, a small $\alpha$ is easily handled with numerical methods and still have a full understanding of all the cosmological solutions of the standard model. This forms the basis of our further work in cosmology that 
is discussed in the following sections.

For further discussion, a useful gauge choice is $\phi(x)=\phi_{0}$ for all spacetime as in Eq.(7)). This is the gauge called the $c$-gauge in our previous work. Because we use also other gauges, we attach the letter $c$ to each field in this gauge, thus $\phi_{c}, s_{c}, g_{c}^{\mu \nu}$ will remind us that we are in the $c$-gauge, where $\phi_{c}(x)=\phi_{0}$. In c-gauge we rename $s_{c}(x)=h(x)$ to recall that in this gauge we obtain the simplest connection to the Higgs field $h(x)$ at low energy, in nearly flat spacetime, $g_{c}^{\mu \nu}=\eta^{\mu \nu}+\cdots$, as discussed in the paragraphs before Eq.(9). The Lagrangian in Eq.(19) with general $u, \bar{G}, f$, takes the following greatly simplified form in the $c$-gauge

$$
\mathcal{L}(x)=\sqrt{-g_{c}}\left[\frac{1}{12} \phi_{0}^{2} u\left(h / \phi_{0}\right) R\left(g_{c}\right)-\frac{1}{2} \bar{G}\left(h / \phi_{0}\right) g_{c}^{\mu \nu} \partial_{\mu} h \partial_{\nu} h-\phi_{0}^{4} f\left(h / \phi_{0}\right)\right] .
$$

If we apply a Weyl transformation to go to the Einstein frame ${ }^{3}, g_{\mu \nu}^{E}=u\left(h / \phi_{0}\right) g_{\mu \nu}^{c}$, then we obtain

$$
\mathcal{L}(x)=\sqrt{-g_{E}}\left[\frac{1}{12} \phi_{0}^{2} R\left(g_{E}\right)-\frac{1}{2} \frac{\left(\partial_{h / \phi_{0}} \sqrt{u}\right)^{2}+\bar{G}\left(h / \phi_{0}\right)}{u\left(h / \phi_{0}\right)} g_{E}^{\mu \nu} \partial_{\mu} h \partial_{\nu} h-\phi_{0}^{4} \frac{f\left(h / \phi_{0}\right)}{\left(u\left(h / \phi_{0}\right)\right)^{2}}\right]
$$

Without loss of generality, a particularly simplifying choice for $\bar{G}\left(h / \phi_{0}\right)$, namely

$$
\bar{G}\left(h / \phi_{0}\right)=u\left(h / \phi_{0}\right)-\left(\partial_{h / \phi_{0}} \sqrt{u}\right)^{2},
$$

is the one that yields a canonically normalized Higgs field. In this form, the theory can be interpreted directly as an Einstein frame formulation of the standard model with a canonically normalized Higgs field ${ }^{4}$ and an effective Higgs potential $V_{\text {eff }}(h)$ given by

$$
V_{\text {eff }}(h)=\phi_{0}^{4} \frac{f\left(h / \phi_{0}\right)}{\left(u\left(h / \phi_{0}\right)\right)^{2}}
$$

This potential can be crafted by various choices of $f(z)$ and $u(z)$ to fit cosmological observations, while still being consistent with an underlying local conformal symmetry. However, since this gauge led to an overall positive coefficient in front of the Ricci scalar, it could

${ }^{3}$ The Weyl transformation is $\sqrt{-g_{c}} u R\left(g_{c}\right)=\sqrt{-g_{E}} R\left(g_{\mu \nu}^{E}\right)+6 \sqrt{u} \partial_{\mu}\left(\sqrt{-g_{E}} g_{E}^{\mu \nu} \partial_{\nu} \sqrt{u}\right)$. After an integration by parts of the last term (or by dropping a total derivative) we obtain the given result.

${ }^{4}$ Alternatively, without fixing $\bar{G}$, it is possible to do a field redefinition such that $h$ is written in terms of a canonically normalized field $\sigma$, where the relation between $\sigma$ and $h(\sigma)$ is given by the first order differential equation $\left(\frac{d h}{d \sigma}\right)^{2}\left[\left(\partial_{h / \phi_{0}} \sqrt{u}\right)^{2}+\bar{G}\left(h / \phi_{0}\right)\right]=u\left(h / \phi_{0}\right)$. Then the Lagrangian rewritten in terms of the canonically normalized $\sigma$ is again of standard form with the same potential $V_{\text {eff }}(h(\sigma))$ as Eq.(24), except for expressing $h$ in terms of $\sigma$. This complicated procedure is avoided by the simple choice of $G$ in Eq.(23). 
not recover all field configurations that are demanded in the geodesically complete theory; inevitably the generic solutions in this gauge are geodesically incomplete as we have learned in our previous work.

For example, the best fit inflaton potentials based on recent Planck satellite data [5] are "plateau" models" [38]. Using the geodesically incomplete form of the theory in (22) it is possible to construct examples with plateaus at large $h / \phi_{0}$, by choosing $f$ and $u$ such that the potential $V_{\text {eff }}(h)$ is very slowly varying and approaches a constant at large $h$ and by requiring $V_{\text {eff }}(h)$ becomes approximately the familiar Higgs potential needed to fit low energy physics for $h \ll \phi_{0}$.

The Bezrukov-Shaposhnikov (BS) model for Higgs inflation [23] is a special case of our more general form in Eq.(21), namely their proposal

$$
\mathcal{L}^{B S}(x)=\sqrt{-g_{c}}\left[\frac{1}{12}\left(\phi_{0}^{2}+\xi h^{2}\right) R\left(g_{c}\right)-\frac{1}{2} g_{c}^{\mu \nu} \partial_{\mu} h \partial_{\nu} h-\frac{\lambda}{4}\left(h^{2}-\alpha^{2} \phi_{0}^{2}\right)^{2}-\frac{\lambda^{\prime}}{4} \phi_{0}^{4}\right],
$$

follows from Eq.(21) by taking $U \rightarrow\left(\phi_{0}^{2}+\xi h^{2}\right), \bar{G} \rightarrow 1$, and $V \rightarrow\left(\frac{\lambda}{4}\left(s^{2}-\alpha^{2} \phi_{0}^{2}\right)^{2}+\frac{\lambda^{\prime}}{4} \phi_{0}^{4}\right)$. This leads to an effective potential $V_{\text {eff }}$ of the type above in Eq.(24),

$$
V_{e f f}(\sigma)=\frac{\frac{\lambda}{4}\left(h^{2}-\alpha^{2} \phi_{0}^{2}\right)^{2}+\frac{\lambda^{\prime}}{4} \phi_{0}^{4}}{\left(1+\xi h^{2} / \phi_{0}^{2}\right)^{2}}, \text { with } h \rightarrow h(\sigma),
$$

In the BS model, because $G$ is chosen as $G=1$, the field $h$ is not canonically normalized, so $h$ must be replaced through a field redefinition [23] to obtain a canonically normalized Higgs, $\sigma$, as described more generally in footnote (4).

Hence, the BS model has a fully Weyl symmetric formulation which was not noticed before. Its presentation in the literature has included various ambiguities and inconsistencies, with clashing ideas on scaling symmetries at the classical level and quantum corrections. For example, in some cases, the coupling to gravity has a dimensionful Newton constant that is inconsistent with the scaling symmetry of the rest of the theory; a massless dilaton is said to exist in cases where global scaling symmetry is explicitly broken at low energies; unimodular gravity has been introduced but this is inconsistent with scale symmetry; and there is ambiguity about which renormalization scheme is appropriate for computing quantum corrections. These issues are fully resolved with the underlying Weyl symmetric formulation discussed here. Also, now that we have recast the BS model into a fully conformally invariant form, we can see in the gauge fixed Einstein frame (22-24) that it is not unique. Rather, it is just a special case of a larger set of conformally invariant models including a range 
that have similar plateau properties. Furthermore, since $U=\phi^{2}+\xi h^{2}$ is purely positive, it means the fields in the BS model form a basis that is geodesically incomplete, and hence its generic solutions can describe only a subsector of the available field space. We make further comments in Sec. (IV) including the effects of geodesic incompleteness.

\section{B. Supergravity}

We do not know if supersymmetry (SUSY) is a property of nature, but theoretically it is an attractive possibility. Therefore, it is of interest to investigate whether it is compatible with an underlying local conformal symmetry. As a superconformal local symmetry, the generalization of the Weyl invariant formalism of the previous section produces stronger constraints on scalar fields. This was derived in [18] from the gauge symmetry formalism in $4+2$ dimensions. It is possible to arrive at the results given below directly in $3+1$ dimensions by requiring supergravity with a local superconformal symmetry, but provided the usual Einstein-Hilbert term is dropped (which is unusual in the supergravity literature), and instead a Weyl symmetric formulation like the previous sections is implemented. In the $4+2$ dimensional approach of $2 \mathrm{~T}$-physics there is no option: it is a prediction that the emergent $3+1$ dimensional theory is automatically invariant under a local symmetry $\mathrm{SU}(2,2 \mid 1)$, where $\mathrm{SU}(2,2)=\mathrm{SO}(4,2)$ is the connection to $4+2$ dimensions, the subgroup $\mathrm{SO}(1,1) \subset \mathrm{SO}(3,1) \times \mathrm{SO}(1,1) \subset \mathrm{SO}(4,2)$ is the Weyl subgroup that acts on the extra $1+1$ dimensions, and the supersymmetrization in $4+2$ dimensions promotes $\mathrm{SU}(2,2)$ to $\mathrm{SU}(2,2 \mid \mathcal{N})$ for $\mathcal{N}$ supersymmetries [17], with $\mathcal{N}=1$ in the present case [18].

The scalar-field sector of the emergent $3+1$ dimensional locally superconformal theory is presented here briefly in a streamlined fashion, including some simplifications and extensions. First note that a scalar field in a chiral supermultiplet must be complex. Thus SUSY requires the singlet $\phi$ to be complexified so that it is a member of a supermultiplet. Then to describe all the scalar fields we use a complex basis $\phi^{m}$ and denote their complex conjugates as $\bar{\phi}^{\bar{m}}$ with the barred index $\bar{m}$. The notation is reminiscent of standard supergravity as reviewed in [40]. However now there is no Einstein-Hilbert term; instead there is a Weyl plus an additional $U(1)$ gauge symmetry that can gauge fix a complex scalar $\phi$ into a dimensionful real constant $\phi_{0}$ that plays the role of the Planck scale.

Recalling the discussion about removing the (real) ghost scalar field $\phi$ in Sec. IIB, it 
should be noticed that the complex $\phi$ amounts to two real ghosts, and therefore two gauge symmetries are needed to remove them. This role is played by the Weyl symmetry $\mathrm{SO}(1,1)$ that acts on the extra $1+1$ dimensions and the local R-symmetry $\mathrm{U}(1)$, both of which are included in the local $\mathrm{SU}(2,2 \mid 1)$ outlined above. The local $\mathrm{U}(1)$ is a crucial ingredient as a partner of the Weyl symmetry to remove the additional ghost from a complexified dilaton field $\phi$ that is demanded by SUSY.

Like before, we have $U(\phi, \bar{\phi})$, but SUSY requires that the metric $G_{m \bar{n}}$ be derived from the derivatives of $U(\phi, \bar{\phi})$ like a Kähler metric

$$
G_{m \bar{n}}=\frac{\partial^{2} U(\phi, \bar{\phi})}{\partial \phi^{m} \partial \phi^{\bar{n}}}
$$

The metric must be non-singular and $\left(-G_{m \bar{n}}\right)$ cannot contain any more than one negative eigenvalue (because no more than one complex ghost can be removed). We denote its inverse formally as $G^{\bar{n} m}=\left(\frac{\partial \bar{\phi} \otimes \partial \phi}{\partial^{2} U}\right)^{\bar{n} m}$. A simple quadratic example similar to Eq.(15) is

$$
U(\phi, \bar{\phi})=\phi^{m} \bar{\phi}^{\bar{n}} \eta_{m \bar{n}}, G_{m \bar{n}}=\eta_{m \bar{n}} \text {, where } \eta_{m \bar{n}}=\operatorname{diag}(1,-1, \cdots,-1) \text {. }
$$

In this example, all scalars in the theory are conformally coupled, and U and G are automatically invariant under a global $\mathrm{SU}(1, \mathrm{~N})$ symmetry. This global symmetry, which will continue to be a hidden symmetry in some gauges, may be broken explicitly by some terms in the potential $V$, depending on the model considered. Nevertheless, keeping track of this (broken) symmetry in physical applications can be useful.

The scalar field sector of the supergravity theory with local Weyl symmetry is given as follows.

$$
\mathcal{L}_{\text {bose }}=\sqrt{-g}\left[\frac{1}{6} U(\phi, \bar{\phi}) R(g)+\frac{\partial^{2} U}{\partial \phi^{m} \partial \bar{\phi}^{n}} g^{\mu \nu} D_{\mu} \phi^{m} D_{\nu} \bar{\phi}^{n}-V_{F+D}(\phi, \bar{\phi})\right] .
$$

The Kähler metric and Kähler potential structure is reminiscent of general supergravity [40], however the absence of the Einstein-Hilbert term, and the corresponding scale invariance is the important difference. Here, $U(\phi, \bar{\phi})$ is homogeneous of degree two and satisfies homothety constraints similar to Eq.(11),

$$
U(t \phi, t \bar{\phi})=t^{2} U(\phi, \bar{\phi}), \frac{\partial U(\phi, \bar{\phi})}{\partial \bar{\phi}^{\bar{m}}}=\phi^{n} \frac{\partial^{2} U(\phi, \bar{\phi})}{\partial \phi^{n} \partial \phi^{\bar{m}}} \text {, and complex conjugate. }
$$

The potential energy has two parts $V_{F+D}(\phi, \bar{\phi})=V_{F}(\phi, \bar{\phi})+V_{D}(\phi, \bar{\phi})$. The potential $V_{F}(\phi, \bar{\phi})$ must be derived from an analytic superpotential $f(\phi)$ that depends only on $\phi^{m}$ 
and not on $\bar{\phi}^{\bar{m}}$, and is homogeneous of degree three so that $V_{F}$ is homogeneous of degree four

$$
f(t \phi)=t^{3} f(\phi), \quad V_{F}=-\left(\frac{\partial \bar{\phi} \otimes \partial \phi}{\partial^{2} U}\right)^{\bar{n} m} \frac{\partial \bar{f}}{\partial \bar{\phi}^{\bar{n}}} \frac{\partial f}{\partial \phi^{m}} .
$$

The potential $V_{D}(\phi, \bar{\phi})$ is derived from another independent analytic function $z_{a b}(\phi)$ with adjoint group indices $a, b$ that appears in Yang-Mills terms as, $-\frac{1}{4} \operatorname{Re}\left(z_{a b}(\phi)\right) F_{\mu \nu}^{a} F^{b \mu \nu}$. This $z_{a b}(\phi)$ should be homogeneous of degree zero $z_{a b}(t \phi)=z_{a b}(\phi)$. Then $V_{D}$ takes the form

$$
V_{D}(\phi, \bar{\phi})=\frac{1}{2} \operatorname{Re}\left(z_{a b}^{-1}\right)\left(\frac{\partial U}{\partial \phi^{m}}\left(t_{a} \phi\right)^{m}\right)\left(\frac{\partial U}{\partial \bar{\phi}^{\bar{n}}}\left(t_{b} \bar{\phi}\right)^{\bar{n}}\right)
$$

where $t_{a}$ is the matrix representation of the Yang-Mills group as it acts on the scalars $\left(\phi^{m}, \bar{\phi}^{\bar{m}}\right)$. The fermionic terms are added consistently with the usual rules of supergravity [40].

This is the general setup for the Weyl invariant matter coupled to Weyl invariant supergravity as derived from the $4+2$ dimensional theory. The simplest example that corresponds to the supersymmetric generalization of Eq.(15) would be the minimal supersymmetric standard model extended with an additional singlet supermultiplet, whose scalar component is the complex field $\phi$. Then $U$ takes the quadratic form suggested in [18]

$$
U=\bar{\phi} \phi-H_{u}^{\dagger} H_{u}-H_{d}^{\dagger} H_{d}
$$

where $H_{u}, H_{d}$ are the two Higgs doublets needed in the supersymmetric version of the standard model. The superpotential $f\left(\phi, H_{d}, H_{u}\right)$ and the matrix $z_{a b}\left(\phi, H_{d}, H_{u}\right)$ are chosen to fit the usual practice of supersymmetric model building [40]. An example is $z_{a b}=\delta_{a b}$, and $f\left(\phi, H_{d}, H_{u}\right)=g\left(H_{u} H_{d}\right) \phi+g^{\prime 3}$, where $\left(H_{u} H_{d}\right)=H_{u}^{\alpha} H_{d}^{\beta} \varepsilon_{\alpha \beta}$ is the only $\mathrm{SU}(2) \times \mathrm{U}(1)$ invariant which is analytic in both $H_{u}$ and $H_{d}$. In an effective (rather than renormalizable) low energy theory, these can be modified by replacing the dimensionless coupling constants $g, g^{\prime}$ by an arbitrary function of the ratio $\left(H_{u} H_{d}\right) / \phi^{2}$, and similarly for $z_{a b}$. When the complex $\phi(x)$ is gauge fixed to the real constant $\phi_{0}$, this approach generates all the dimensionful parameters from the same source $\phi_{0}$ which is of order of the Planck scale. Then we see that the modification of $g, g^{\prime}$ and $z_{a b}$ by arbitrary functions of $\left(H_{u} H_{d}\right) / \phi^{2}$ is negligible at low energies.

More general scale invariant models with more fields (such as generalizations of the minimal SUSY model) are easily constructed by using the rules on scalar fields given in this section. With special forms of the superpotential $f(\phi)$, it is possible to construct so called 
"no-scale" models [41]-[43] in which the cosmological constant is guaranteed to be zero at the classical level even after spontaneous breakdown of symmetries. Simple examples of no-scale models, that are lifted to be fully Weyl invariant, are given in [18].

In this paper we will not explore any further the general superconformal structures discussed above although this could be of interest in some future applications. Some of our examples that are very similar to the Weyl invariant supersymmetric cases previously discovered in [18] were later explored in a cosmological context in [28]. However their discussion, which is focussed on very specialized initial conditions that are so non-generic, is difficult to be convincing especially in the face of the complete set of solutions that we now understand much better.

\section{CONFORMAL COSMOLOGY}

In this paper we have emphasized the geodesically complete nature of our conformal standard model. In this section we first describe what we mean by geodesic completeness and the related notion of completeness in field space, and then discuss some applications of our conformal standard model in cosmology.

There are two required properties for geodesic completeness: the first is smooth local geodesic continuation through singularities across all space-time patches; the second is infinite action for geodesics that reach arbitrarily far into the past so that unnatural initial conditions are avoided. Both properties are satisfied in our theory which is insured to also be complete in field space. Geodesics $x^{\mu}(\lambda)$ are computed by extremizing the particle action $S=-\int d \lambda m \sqrt{\dot{x}^{\mu} \dot{x}^{\nu} g_{\mu \nu}(x(\lambda))}$ where $m$ is the mass and $g_{\mu \nu}$ is a gravitational field. In our Weyl invariant standard model (5) all particle masses are generated by the Higgs field, so $m$ is proportional to the Higgs field $h, m=g h(x(\lambda))$, where $g$ is the dimensionless coupling constant for the corresponding particle as prescribed by the standard model. Then this particle action is locally invariant under Weyl transformations $h(x) \rightarrow \Omega(x) h(x)$ and $g_{\mu \nu}(x) \rightarrow \Omega^{-2}(x) g_{\mu \nu}(x)$. For our cosmological discussion, geodesics $x^{\mu}(\lambda)$ in a spatially homogeneous space are computed when both homogeneous fields that appear in the particle action $S, h(\tau)$ and $g_{\mu \nu}(\tau)=a^{2}(\tau)\left(\eta_{\mu \nu}+\right.$ anisotropy \& curvature), where $\tau \equiv x^{0}$ is the conformal time, correspond to consistent solutions of the field equations of our conformal standard model in (5). The full solution to the geodesic equation (neglecting curvature and 
anisotropy for simplicity) is

$$
\vec{x}(\tau)=\vec{q}+\vec{p} \int_{\tau_{0}}^{\tau} \frac{d \tau^{\prime}}{\sqrt{\vec{p}^{2}+m^{2}\left(\tau^{\prime}\right) a^{2}\left(\tau^{\prime}\right)}} .
$$

where $\vec{p}$ is the conserved spatial component of the particle momentum vector, $p^{\mu}=\partial S / \partial \dot{x}^{\mu}$, while $p^{0}=\sqrt{\vec{p}^{2}+m^{2}(\tau) a^{2}(\tau)}$, and $\vec{q}=\vec{x}\left(\tau_{0}\right)$ is the initial position. These expressions are invariant under reparametrizations of the affine parameter $\lambda$, since they depend only on the physical spacetime variables $x^{\mu}$. Then, the local continuation property of a geodesic $\vec{x}(\tau)$ in a homogeneous space is satisfied automatically when one insures that all the fields, including $m(\tau)=g h(\tau)$ and $a(\tau)$, are given in all the patches of homogeneous field space and that the fields are smoothly continued through singularities. In [10] [11], we showed how local Weyl invariance, in the special Weyl frame displayed in Eq.(5) that covers all the patches, are essential ingredients to insure that all homogeneous cosmological solutions of our conformal standard model (5) have this continuation property.

The global action for a geodesic (34) is given by

$$
\left|S_{f i}\right|=\int_{\tau_{i}}^{\tau_{f}} d \tau \frac{m^{2}(\tau) a^{2}(\tau)}{\sqrt{\vec{p}^{2}+m^{2}(\tau) a^{2}(\tau)}} .
$$

The combination $m^{2}(\tau) a^{2}(\tau)=g^{2} h^{2}(\tau) a^{2}(\tau)$ that appears in $\left|S_{f i}\right|$ is invariant under Weyl transformations, so it can be computed in any convenient gauge (see e.g. Eqs.(36-38)) . In particular in the $\gamma$-gauge used in the solutions in [10] [11], where $a_{\gamma}(\tau)=1$, we have shown that in all cyclic solutions in [11], $h^{2}(\tau) a^{2}(\tau)=h_{\gamma}^{2}(\tau) a_{\gamma}^{2}(\tau)$ oscillates with a fixed maximum amplitude many times within a cycle, and for an infinite number of times over an infinite number of cycles, leading to an infinite action, since $\left|S_{f i}\right|$ receives the same positive finite contribution in each cycle. For these solutions the average magnitude of $\left|a_{E}\right|$ in the E-gauge (usual Einstein frame, see Eq.(36) ) is the same in each cycle. Recently, we have also constructed [27] solutions with increasing entropy in each future cycle such that the average $\left|a_{E}\right|$ in the $E$-gauge increases in each future cycle. For these solutions, $\left|S_{f i}\right|$ diverges to the past [27] even faster as compared to our solutions in [11]. Hence, unlike inflation scenarios [44], we may expect that the universe described by our conformal standard model has no unnatural initial conditions.

Next, we discuss various potential applications of Weyl invariant theories to cosmology. Early universe cosmology near the singularity is a natural place to look for uses because the interactions imposed by Weyl invariance (more profoundly $4+2$ dimensional gauge 
symmetries) produce their most significant changes relative to conventional physics in the limit of strong gravity, as noted in some of the examples above. Black hole phenomena is another interesting arena for study, but we will not discuss them here.

The first important application was to use Weyl invariance to construct geodesically complete cosmological solutions. Conventional cosmological analysis is usually confined to theories coupled to Einstein gravity with a dimensionful Newton's constant. In this framework, all cosmological solutions of interest are geodesically incomplete. However, we have observed that a Weyl-invariant theory can be cast in Einstein frame, as illustrated by Eq.(22), and in other frames. The observation made in [8]-[12] is that geodesically incompleteness is an artifact of an unsuitable frame choice: geodesically incomplete solutions in Einstein frame may be completed in other frames, even though the theories are entirely equivalent away from the singularity.

The Einstein frame can always be reached directly from a Weyl invariant theory in (19) by making the Einstein-gauge choice

$$
\frac{1}{12} U\left(\phi_{E}, s_{E}\right)=(16 \pi G)^{-1}=\frac{1}{2} .
$$

We label this the $E$-gauge and mark the fields in this gauge with the subscript $E$ (as in $\left.\left(\phi_{E}, s_{E}, g_{E}^{\mu \nu}\right)\right)$ to distinguish them from the $c$ - gauge fields $\left(\phi_{c}, s_{c}, g_{c}^{\mu \nu}\right)$. Note that the $E$ gauge can be valid only in a patch in field space since restrictions must be imposed on the fields to require that $U\left(\phi_{E}, s_{E}\right)$ is positive.

The same theory can easily be transformed to other gauges. For example, the relations between the $E$ - and $c$-gauge fields can be easily derived by considering the Weyl gauge invariants such as $s / \phi$ and $(\operatorname{det}(-g))^{1 / 8} \phi,(\operatorname{det}(-g))^{1 / 8} s$, etc., for example we deduce

$$
\frac{s_{E}}{\phi_{E}}=\frac{h}{\phi_{0}}, \text { and }\left(\operatorname{det}\left(-g_{c}\right)\right)^{1 / 8} \phi_{0}=\left(\operatorname{det}\left(-g_{E}\right)\right)^{1 / 8} \phi_{E} \text {, etc. }
$$

From these, we can express $\phi_{E}(h)$ and $s_{E}(h)$ in terms of the single field $h$, so that the gauge condition (36) is satisfied. Inserting these expressions into the gauge invariant action (19) we arrive at the same $E$-frame action as Eq.(22).

As argued in our work [8]-[12], classically geodesically complete solutions can be obtained for all single-scalar theories that can be cast in the form of Eq.(19). But, for all patches of field space $\left(\phi, s, g_{\mu \nu}\right)$ to be included, as demanded by the geodesics derived from $V_{\text {eff }}(h)$ in the Einstein frame, $(U, G, f)$ must be brought to an appropriate form by using the field 
redefinitions discussed below Eq.(20) . The patches of field space $\left(\phi, s, g_{\mu \nu}\right)$ that are missing in the Einstein frame can then be added in order to obtain a geodesically complete space. We have argued in [10], and provided a proof in section (IIIA), that geodesic completeness is accomplished when we bring $U$ to the form $U=\phi^{2}-s^{2}$, where $s^{2}=\sum s_{I}^{2}$, is the sum of all scalars other than $\phi$.

Geodesics remain incomplete when they hit the singularity in all frames in which $U$ is always positive, or the equivalent Einstein frame, such as the one in Eq.(25). When we rewrite those, by field redefinitions, in terms of new fields in which $U=\phi^{2}-s^{2}$, then the patches of field space $\phi^{2} \geq s^{2}$ are equivalent to the Einstein frame, or to the other frames with positive $U$. Geodesic completion is achieved by allowing all regions of field space in the parametrization that has $U=\phi^{2}-s^{2}$; for this form, $U$ is allowed to smoothly go negative. In these coordinates the gauge invariant vanishing point of $U=0$, given by the gauge invariant expression $|s / \phi|=1$, has a special significance; it corresponds to the singularity of the scale factor of the universe in the Einstein gauge. This is seen by equating the gauge invariant $(\operatorname{det}(g))^{1 / 4} U(\phi, s)$ in the Einstein gauge, in which $U\left(\phi_{E}, s_{E}\right)=6$, and the unimodular gauge (labelled with $\gamma$ ), in which $\operatorname{det}\left(-g_{\gamma}\right)=1$, as follows

$$
(\operatorname{det}(-g))^{1 / 4} U(\phi, s)=\left(\operatorname{det}\left(-g_{E}\right)\right)^{1 / 4} \times 6=1 \times U\left(\phi_{\gamma}, s_{\gamma}\right) .
$$

At spacetime points or regions where $U\left(\phi_{\gamma}, s_{\gamma}\right)=\phi_{\gamma}^{2}-s_{\gamma}^{2}=0$, which is where the gauge invariant quantity $|s / \phi|$ hits unity in all gauges, $\left|s_{\gamma} / \phi_{\gamma}\right|=\left|s_{E} / \phi_{E}\right|=\left|h / \phi_{0}\right|=1$, the geometry in the Einstein gauge fails completely since det $\left(-g_{E}\right)=0$, and this is how the cosmological singularity occurs at some point in time [10]. Then, as we can see in the $c$ gauge, the region $h / \phi_{0} \sim 1$ (Higgs of Planck size) is the region of the big crunch or big bang singularity, where $|s / \phi|=1$ in any gauge. The behavior of the universe in this region is governed by a universal attractor mechanism that is independent of the scalar potential, therefore independent of the details of the model [10].

Although in the classical theory, cosmological singularities of FRW-type are typically resolved in the Weyl-lifted theory with $U=\phi^{2}-s^{2}$ in suitable Weyl gauges, one should still worry about quantum gravity corrections. When $U$ vanishes, the coefficient of the Ricci scalar in the gravitational action vanishes so there is no suppression of metric fluctuations and quantum gravity corrections should become large. However, it is notable that for certain types of cosmic singularities, including realistic ones, the metric and fields possess a unique 
continuation around the singularity in the complex time plane. A complex time path can be chosen to remain far from the singularity so that $U$ is always large and gravity remains weak. Thus, we are able to find an analytic continuation of our classical solutions connecting big crunches to big bangs along which quantum gravity corrections are small. This issue is under active investigation and we defer further discussion to future work.

A particularly interesting application of Weyl-invariance is to Higgs cosmology, which the geodesically complete solutions show can be much more interesting than conventionally assumed. In fact, there is the possibility that the Higgs field alone may be sufficient to explain the large-scale features of the universe, as suggested by the Bezrukov-Shaposhnikov Higgs inflation model [23] and our recent work on the Higgs in cyclic cosmology [26] [27].

In considering Higgs cosmology or other models of the early universe, we believe that the cosmological solutions found for geodesically complete theories provide some important new insights on the question of the likely initial conditions just after the big bang. For example, the generic behavior of the Higgs after the bang when $h \sim \phi_{0}$ is dramatically different than the contrived initial conditions that are commonly assumed in Higgs inflation scenarios. This is easily seen by examining the analytic work in [10] [11], where for the simple model in Eq.(5) with $\alpha=0$, all homogeneous solutions including curvature and radiation are obtained and the effects of anisotropy are determined [10]. Emphasizing that this is not only the contrived solutions, but all solutions, it serves as an example of the richness of the phenomena that occur in a model even with a simple potential. The results make clear a point that is obvious, but often forgotten: for a given scalar potential, there is an enormous range of cosmological solutions. By comparison, it is clear that the slow-roll initial conditions frequently assumed in the analysis of cosmic inflation are very special and unlikely. For example, by transforming the inflationary solution in the Bezrukov-Shaposhnikov Higgs inflation model [23] to a field basis in which $U=\phi^{2}-s^{2}$, it may be possible to trace cosmic evolution right back to the singularity and to judge whether the inflation is likely in the space of geodesically complete cosmological solutions.

Fig.(1) is an illustration of our solution for the generic cosmological behavior of the Higgs field just after the big bang if the Higgs potential has a stable non-trivial minimum, as in Eq.(5), as usually assumed and as required for the Bezrukov-Shaposhnikov Higgs inflation model [23]. This figure describes the generic cosmological evolution of the Higgs field, that must start with fluctuations of Planck size and energy (due to the universal attractor near 
the singularity [10]), and quickly reduce its amplitude by losing energy to the gravitational field; then after a phase transition, settle down to an almost constant value at the electroweak scale $v$ determined by the dimensionless parameter $\alpha$ in Eq.(3).

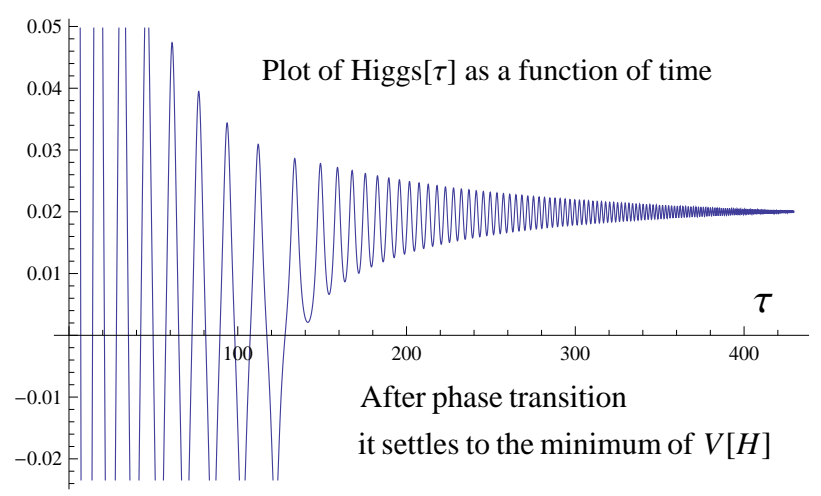

Fig.(1) - After the Big Bang the Higgs field oscillates initially around zero with a large amplitude of the order of the Planck scale. It slowly loses energy to the gravitational field, causing its amplitude to diminish. As it approaches the time or energies of the electroweak scale, it undergoes the phase transition seen in the figure, and then slowly settles to a constant vacuum value $v$ at a stable minimum of the potential.

The solution of Fig.(1) changes drastically if the vacuum is metastable after including quantum corrections, which is a possibility suggested by the most recent LHC data for the Higgs and the top quark masses [19], and assuming no new physics up to the Planck scale. Metastability is incompatible with Higgs inflation and generally causes problems for inflation because the Higgs will typically escape from the metastable phase right after the big bang and roll to a state of negative energy density that can prevent inflation of any sort from occurring.

The exact solutions of the Weyl-invariant theory suggest an alternative cosmology in this case. The generic solution at first behaves as in Fig.(1) after the big bang, all the way through the electroweak phase transition. But after some time (order of the lifetime of the universe) the Higgs oscillations in the electroweak vacuum grow larger and larger, like the mirror image of Fig.(1), taking away energy from the gravitational field and eventually causing a collapse of the universe to a big crunch, while the Higgs does a quantum tunneling to a lower state of the potential. At that stage our exact analysis near the singularity given in [10] takes over to describe interesting new phenomena that occur just after the crunch and before another rebirth of the universe with a big bang. The result is a regularly 
repeating sequence in which the Higgs field is trapped in its metastable state after a big bang, remains there for a long period of expansion followed by contraction, escapes as the universe approaches the big crunch, passes through to a big bang and becomes trapped again. The evolution can be considered a Higgs-driven cyclic theory of the universe. The details are presented in a separate paper [27].

\section{Acknowledgments}

This research was partially supported by the U.S. Department of Energy under grant number DE-FG03-84ER40168 (IB) and under grant number DE-FG02- 91ER40671 (PJS).

Research at Perimeter Institute is supported by the Government of Canada through Industry Canada and by the Province of Ontario through the Ministry of Research and Innovation. The work of NT is supported in part by a grant from the John Templeton Foundation. The opinions expressed in this publication are those of the authors and do not necessarily reflect the views of the John Templeton Foundation. IB thanks CERN and PJS thanks Perimeter Institute for hospitality while this research was completed.

[1] P.A.M. Dirac, Ann. Math. 37,429 (1936); G. Mack and A. Salam, Ann. Phys. (N.Y.) 53, 174 (1969).

[2] H. Weyl, "Spacetime Matter" (Dover, NY, 1922), sect.35; Sitzungsber. Preuss Acad. d. Wissensch (1918) 465, reprinted in The Principles of Relativity (Dover, NY, 1923).

[3] S. Deser, "Scale Invariance and Gravitational Coupling", Ann.Phys. 59 (1970) 248.

[4] F. Englert, E. Gunzig, C. Truffin, P. Windey, "Conformal Invariant General Relativity with Dynamical Symmetry Breakdown," Phys.Lett. B57 (1975) 73; F. Englert, C. Truffin and R. Gastmans, "Conformal Invariance in Quantum Gravity", Nuclear Physics B117 (1976) 407.

[5] P. Ade et al. (Planck Collaboration), arXiv: 1303.5082.

[6] J. L. Sievers, R. A. Hlozek, M. R. Nolta, V. Acquaviva, G. E. Addison, et al. arXiv: 1301.0824.

[7] I. Bars and S-H. Chen, "The Big Bang and Inflation United by an Analytic Solution ", Pays. Rev. D83 043522 (2011) arXiv:1004.0752].

[8] I. Bars, S-H. Chen and N. Turok, "Geodesically Complete Analytic Solutions for a Cyclic 
Universe", Phys. Rev. D84 083513 (2011) arXiv:1105.3606].

[9] I. Bars, "Geodesically Complete Universe", arXiv:1109.5872, in Proceedings of the DPF- 2011 Conference, Providence, RI, August 8-13, 2011, http://www.slac.stanford.edu/econf/C110809/.

[10] I. Bars, S.-H. Chen, P. J. Steinhardt and N. Turok, "Antigravity and the Big Crunch/Big Bang Transition", Phys. Lett. B715 (2012) 278-281 [arXiv:1112.2470].

[11] I. Bars, S.-H. Chen, P. J. Steinhardt and N. Turok, "Complete Set of Homogeneous Isotropic Analytic Solutions in Scalar-Tensor Cosmology with Radiation and Curvature", Phys. Rev. D86 (2012) 083542 [ arXiv:1207.1940].

[12] I. Bars, "Traversing Cosmological Singularities, Complete Journeys Through Spacetime Including Antigravity", arXiv:1209.1068, in Beyond the Big Bang, Competing Scenarios for an Eternal Universe, Ed. Rudy Vaas, Springer [2013], ISBN 978-3-540-71422-4.

[13] I. Bars, "Gauge Symmetry in Phase Space, Consequences for Physics and Spacetime", Int. J. Mod. Phys. A25 (2010) 5235 [arXiv:1004.0668].

[14] I. Bars, "The Standard Model of Particles and Forces in the Framework of 2T-physics", Phys. Rev. D74 (2006) 085019 hep-th/0606045.

[15] I. Bars "The Standard model as a 2T- physics theory", AIP Conf.Proc. 903 (2007) 550 [hepth/0610187].

[16] I. Bars, "Gravity in 2T-Physics", Phys. Rev. D77 (2008) 125027 arXiv:0804.1585]; I. Bars, S. H. Chen "Geometry and Symmetry Structures in 2T Gravity", Phys. Rev. D79 (2009) 085021 arXiv:0811.2510v2].

[17] I. Bars and Y-C Kuo, "Field Theory in 2T-physics with $\mathcal{N}=1$ Supersymmetry", Phys. Rev. Lett. 99 (2007) 041801 hep-th/0702089]; "Supersymmetric Field Theory in 2T-physics ", Phys. Rev. D76 (2007) 105028 [ hep-th/0703002]; “N=2,4 Supersymmetric Gauge Field Theory in 2T-physics", Phys. Rev. D79 (2009) 025001 arXiv:0808.0537]; "Super Yang-Mills theory in 10+2 dimensions, The 2T-physics Source for N=4 SYM and M(atrix) Theory", arXiv:1008.4761.

[18] I. Bars, "Constraints on Interacting Scalars in 2T Field Theory and No Scale Models in $1 \mathrm{~T}$ Field Theory", Phys. Rev. D82 (2010) 125025 arXiv:1008.1540].

[19] G. Degrassi, S. Di Vita, J. Elias-Miró, J. R. Espinosa, G.F. Giudice, G. Isidori, A. Strumia, "Higgs mass and vacuum stability in the Standard Model at NNLO", arXiv:1205.6497. 
[20] W.A. Bardeen, "On Naturalness in the Standard Model", preprint FERMILAB-CONF-95391-T; "Mechanisms of Electroweak Symmetry Breaking and the Role of a Heavy Top Quark", preprint FERMILABCONF-95-377-T.

[21] K. Meissner and H. Nicolai, "Conformal Symmetry and the Standard Model", Phys. Lett. B648 (2007) 312, arXiv:hep-th/0612165. "Effective action, conformal anomaly and the issue of quadratic divergences", Phys.Lett. B660 (2008) 260 [arXiv:0710.2840].

[22] A. Codello, G. D’Odorico, C. Pagani, R. Percacci, "The Renormalization Group and Weylinvariance," Class. Quant. Grav. 30 (2013) 115015 arXiv:1210.3284.

[23] F. L. Bezrukov and M. Shaposhnikov, "The Standard Model Higgs boson as the inflaton", Phys. Lett. B659 (2008) 703 [arXiv:0710.3755].

[24] P. J. Steinhardt and N. Turok, Science 296, 1436 (2002).

[25] P. J. Steinhardt and N. Turok, Phys.Rev. D65, 126003 (2002), hep-th/0111098.

[26] Itzhak Bars, at conference on "Conformal Nature of the Universe", Perimeter Institute, May 2012; Neil Turok, CERN colloquium, July 2012; T.W.B. Kibble 80th birthday conference, Imperial College, March 2013; Yale physics department colloquium, April 2013; Paul Steinhardt, at Davis Conference, May 2013.

[27] I. Bars, P. J. Steinhardt and N. Turok, "Cyclic Cosmology, Conformal Symmetry and the Metastability of the Higgs," Phys. Lett. B726 (2013) 50 [arXiv:1307.8106].

[28] S. Ferrara, R. Kallosh, A. Linde, A. Marrani and A. Van Proeyen, "Superconformal Symmetry, NMSSM, and Inflation", Phys. Rev. D83 (2011) 025008 arXiv:1008.2942]; R. Kallosh and A. Linde "Superconformal Generalization of the Chaotic Inflation Model" arXiv:1306.3211 and "Superconformal generalizations of the Starobinsky model", arXiv:1306.3214,

[29] I. Bars, S-H Chen and G. Quelin, "Dual field theories in $(d-1)+1$ emergent spacetimes from a unifying field theory in d+2 spacetime", Phys. Rev. D76 (2007) 065016 arXiv:0705.2834]; I. Bars and G. Quelin, "Dualities among one-time field theories with spin, emerging from a unifying two- time field theory", Phys. Rev. D77 (2008) 125019 [arXiv:0802.1947].

[30] M. Shaposhnikov, "Is there new physics between electroweak and Planck scales?", arXiv:0708.3550.

[31] W. D. Goldberger, B. Grinstein and W. Skiba, "Light scalar at LHC: The Higgs or the dilaton?", arXiv:0708.1463.

[32] R. Foot, A. Kobakhidze and R. R. Volkas, "Electroweak Higgs as a pseudo-Goldstone bo- 
son of broken scale invariance," Phys. Lett. B655 (2007) 156 arXiv:0704.1165. R. Foot, A. Kobakhidze, K. McDonald, R.Volkas,

[33] R. Foot, A. Kobakhidze, K. McDonald, R.Volkas, "Neutrino mass in radiatively-broken scaleinvariant models", arXiv:0706.1829; "A solution to the hierarchy problem from an almost decoupled hidden sector within a classically scale invariant theory", arXiv:0709.2750.

[34] J. R. Espinosa and M. Quiros, "Novel Effects in Electroweak Breaking from a Hidden Sector", Phys.Rev.D76 (2007) 076004 arXiv:hep-ph/0701145.

[35] G. tHooft. Lecture in conference, Conformal Nature of the Universe, see http://www.pirsa.org/C12027.

[36] G. Belanger, U, Ellwanger, J. F. Gunion, Y. Jiang, S. Kraml, and J. H. Schwarz, "Higgs Bosons at 98 and $125 \mathrm{GeV}$ at LEP and the LHC", JHEP 1301 (2013) 069 [arXiv:1210.1976].

[37] H. Cheng, "The Possible Existence Of Weyl's Vector Meson," Phys. Rev. Lett. 61 (1988) 2182; H. Nishino and S. Rajpoot, "Broken Scale Invariance in the Standard Model", hep-th/0403039. ibid. Class. \& Quant. Gr. 28 (2011) 145014; AIP Conf. Proc. 881 (2007) p. 82.

[38] A. Ijjas, A. Loeb and P.J. Steinhardt, Phys. Lett. B723 (2013) 261.

[39] S. Coleman and E. Weinberg, "Radiative Corrections as the Origin of Spontaneous Symmetry Breaking", Phys. Rev. D7 (1973) 1888.

[40] See for example S. Weinberg, The Quantum Theory of Fields, Volume III, Cambridge 2000.

[41] E. Cremmer, S. Ferrara, C. Kounnas and D. Nanopoulos, "Naturally vanishing cosmological constant in N=1 supergravity", Phys. Lett. 133B (1983) 61.

[42] J. Ellis, C. Kounnas, and D. Nanopoulos, "No scale supersymmetric GUTs", Nucl. Phys. B247 (1984) 373; ibid "No Scale Supergravity Models With A Planck Mass Gravitino," Phys. Lett.B143 (1984) 410.

[43] A. Lahanas and D. Nanopoulos , "The Road to No Scale Supergravity," Phys. Rept. 145 (1987) 1.

[44] A. Borde, A. H. Guth and A. Vilenkin, Phys. Rev. Lett. 90, 151301 (2003) arXiv:gr-qc/0110012. 